\title{
Evaluating Contract Compatibility for Service Composition in The $\mathrm{SeCO}_{2}$ Framework ${ }^{\star}$
}

\author{
Marco Comerio ${ }^{1}$, Hong-Linh Truong ${ }^{2}$, Flavio De Paoli ${ }^{1}$, Schahram Dustdar $^{2}$ \\ 1 University of Milano - Bicocca, Milano, Italy \\ \{comerio, depaoli\}@disco.unimib.it \\ 2 Distributed Systems Group, Vienna University of Technology \\ \{truong, dustdar\}@infosys.tuwien.ac.at
}

\begin{abstract}
Recently, the Software-as-a-Service (SaaS) model has been increasingly supported, becoming a major part of the new emerging cloud computing paradigms. Although SaaS exists in different forms, supporting and providing SaaS developed based Web services has attracted a large effort from industries and academics because this form of SaaS allows software to be easily composed and integrated to offer new services for customers. Even though various service composition techniques, based on functional and non-functional parameters, have been proposed, the issue of service contract compatibility has been neglected. This issue is of paramount importance in the Web services-based SaaS model because services are provided by different providers, associated with different contracts which are defined by different specifications. This paper proposes techniques for supporting service composers to deal with the heterogeneity of service contracts in service composition. We describe a novel approach for modeling and mapping different service contract specifications, and a set of techniques for evaluating service contract compatibility. Our techniques consider contract terms associated with data and control flows, as well as composition patterns. Illustrating scenarios are proposed to demonstrate the efficiency of our techniques.
\end{abstract}

\section{Introduction}

We have recently observed the rise of cloud computing and SaaS as a part of the cloud computing paradigm [1]. In particular, many providers have provided SaaS using the Web services model. This form of SaaS has been widely supported because it enables service composition and integration.

Techniques supporting service composition and integration have been developed for a long time. It is important that when services are selected and composed from different SaaS providers, their contracts, which govern how the services should be used, have to be compatible. We need to support both, users and tools, to deal with issues related to service contracts. This support is of paramount importance because how services are used is bound to the service

\footnotetext{
* This work is partially supported by the European Union through the FP7-216256 project COIN
} 
contract. However, there is a lack of supporting tools to deal with the evaluation of service contract compatibility, which is actually just one of many open questions about the relationship between service contracts and service composition discussed in [2]. Current techniques, such as service license compatibility [3], are not suitable because they assume that service contracts follow the same specification and they do not support service contracts for service compositions. To our best knowledge, until now, there is no work supporting service contract compatibility that takes into account the heterogeneity of service contract specifications and different aspects associated with data and control flows of the composition.

In this paper, we present an overview of $\mathrm{SeCO}_{2}$, a novel framework for supporting service composers to deal with the heterogeneity of service contracts in service compositions. The framework is a part of tools and systems for supporting the life-cycle management of the ecosystem of service contracts. Within this paper we present the following contributions: (i) a novel approach for modeling and mapping different service contract specifications, and (ii) a set of techniques for service contract compatibility evaluation. Our techniques consider contract terms associated with data and control flows, as well as composition patterns.

The rest of this paper is structured as follows: Section 2 elaborates the context, motivation, and related work of this paper. We discuss our approach and give an overview of $\mathrm{SeCO}_{2}$ in Section 3. We present techniques to achieve the modeling and mapping of service contract specifications in Section 4. We present the compatibility evaluation in Section 5. Experiments are presented in Section 6. We conclude the paper and give an outlook to the future work in Section 7.

\section{Motivation and Related Work}

\subsection{Motivation}

The main motivation of our work is, in general, how to ensure the compatibility of service contracts for service compositions. In the current service composition landscape there is the need to compose different services to provide converged services. In the SaaS model it is assumed that the service customer uses the software deployed as a service. This model allows service providers to combine different services, potentially characterizing by different service contracts specified by different languages. With the techniques developed so far, it is not so difficult for consumers to compose different services based on published service interfaces. For example, existing platforms like The Process Factory ${ }^{3}$ and Boomi ${ }^{4}$ provide different connectors for consumers to compose their services from various SaaS providers. However, the consumers need to ensure that the service compositions do not include conflicting service contracts. This assurance cannot be given by a single SaaS provider and currently is not available in existing composition tools.

In the SaaS and cloud computing model, no single specification would be agreed by all, making the service contract compatibility evaluation hard. Past

\footnotetext{
${ }^{3}$ http://www. theprocessfactory.com

${ }^{4}$ http://www. boomi.com
} 
research has neglected contracts of composite services when performing service composition by considering only functional parameters (service interfaces) or assume that contracts associated with services being composed are described by a single language. Furthermore, past research has not focused on tools and algorithms dealing with contract compatibility evaluation when combining different services from different providers. Typically, they deal with only contract negotiation between consumer and service in a point-to-point manner.

Service contract compatibility is also strongly dependent on the structure of composition. This is related to not only control flows but also data flows and composition patterns. While certain works address QoS-based compatibility for control flows, currently there is a lack of a good understanding of how to check contract compatibility for data, the input/output of services, whose contract terms are not always the same to that of the service operations. We stress that contract terms associated with the use of service and the use of data are different and our objective is to address the compatibility for both data and service.

\subsection{Related Work on Service Contract Compatibility}

As stated in [2], the understanding between a service consumer and a service provider can be established using different approaches (e.g., policies, licenses, service level agreements). Even if some philosophical differences exist among these approaches, in this paper we identify them under the common term service contract which specifies conditions that a service consumer and a service provider agree. Besides functional terms, a service contract is composed of the specification of Quality of Service (QoS), Business, Service Context and License terms of a service. $Q o S$ terms (e.g., response time) represent technical aspects of the service. Business terms (e.g., service price) describe financial terms and conditions. Service Context terms (e.g., service delivery location) define the characteristics of the context associated with the service. Finally, License terms (e.g., limitation of liabilities and usage permissions) state responsibilities among involved parties and conditions on service usage.

Currently, service contracts can be described using several specifications, such as ODRL-S [4], WSLA [5], and WSOL [6]. Even though these specifications have some common parts, there exists no reference ontology/thesaurus for describing contract properties. This means that service consumers and providers specify their service contracts as they wish, causing many issues when multiple services governed by different contracts are utilized (e.g., in a composition). Until now we are not aware of any work dealing with the definition of techniques to evaluate the compatibility among contract terms specified in different languages.

The most cited works [7-9] are related to contract-based service composition and reduce the problem to the evaluation of QoS constraints among composite services and user requirements. The AgFlow framework [7] evaluates the QoS of composite services with an extensible multidimensional quality model and considering the control flow of the service composition. Other examples of QoSbased composition are in [8] that aims at defining composition rules to evaluate global values of QoS dimensions according to specific workflow patterns. The 
constraint-driven Web service composition tool presented in [9] reduces the service composition problem to a constraint satisfaction problem focusing on business and process constraints. These works consider only a small set of service contract terms (i.e., QoS). The evaluation of qualitative properties (e.g., license terms) are not tackled. Moreover, they assume that property descriptions are always available and specified using a common language.

\section{Overview of The $\mathrm{SeCO}_{2}$ Framework}

The objective of the $\mathrm{SeCO}_{2}$ framework is to support service composers to deal with the heterogeneity of service contracts in service composition. In this paper we focus on techniques used by $\mathrm{SeCO}_{2}$ to support the modeling and mapping of service contracts defined using different specification languages and the evaluation of the compatibility among these service contracts. Figure 1 provides an overview of actors and data involved in these activities.

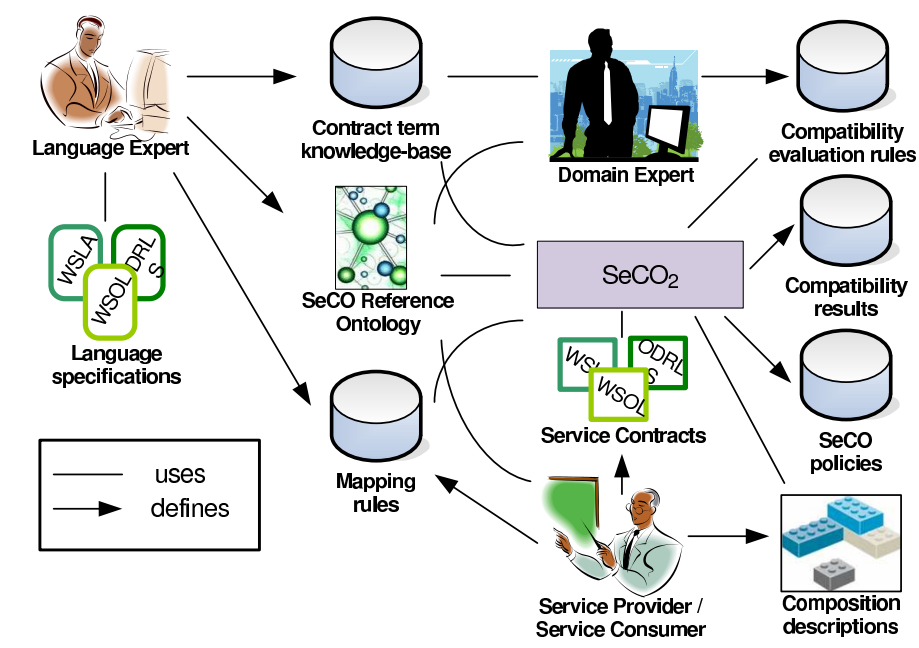

Fig. 1. The $\mathrm{SeCO}_{2}$ Framework

The $\mathrm{SeCO}_{2}$ framework overcomes the heterogeneity in service contract specifications using the SeCO Reference Ontology containing semantic descriptions of service contract properties and the Contract term knowledge-base specifying additional information about these properties. The SeCO Reference Ontology is built based on the Policy-Centered Metamodel (PCM) [10]. The PCM offers (i) the concept of Policy that aggregates property descriptions into a single entity with an applicability condition, and (ii) a set of constraint operators that allows for the description of both qualitative and quantitative properties.

The $\mathrm{SeCO}_{2}$ framework deals with service contracts specified in different languages (e.g., ODRL-S, WSLA and WSOL). In this paper we assume that $\mathrm{SeCO}_{2}$ receives these contracts from service providers and we show how it makes them comparable by wrapping them into SeCO Policies. In order to do this, Language 
Experts analyze language specifications and create, modify, update and delete the knowledge stored in the SeCO Reference Ontology and in the Contract term knowledge-base. The mapping between ontological concepts and contract-specific terminologies is defined by Language Experts, Service Providers and Consumers with mapping rules. In order to define techniques for service contract compatibility evaluation, the $\mathrm{SeCO}_{2}$ framework supports Domain Experts in the definition of compatibility evaluation rules by means of the $\mathrm{SeCO}_{2}$ Reference Ontology and the Contract term knowledge-base. Mapping rules and compatibility evaluation rules, as well as the SeCO Reference Ontology and the Contract term knowledge-base, are shared information for the users. This aspect reduces the effort for their definition and improves reusability. These rules allow $\mathrm{SeCO}_{2}$ to receive Service contracts and Composition descriptions as inputs, perform the wrapping to $\mathrm{SeCO}$ policies and verify the compatibility among them.

One of the most innovative characteristics of our framework is how the compatibility evaluation is performed. Currently, there is no distinction between the description of properties related to service usage (e.g., Request Limit) and properties related to the data produced by the service (e.g., Data Ownership). This produces ambiguities in service contract specifications. As an example, the property Price can refer to the amount of money needed for invoking a service or it can refer to the amount of money needed for receiving an amount of data from a service. This distinction is critical for the service contract compatibility evaluation. The $\mathrm{SeCO}_{2}$ framework performs the compatibility evaluation considering both the control flow and the data flow of the service composition. Dependencies between each service contract property and control and data flow are identified and considered during the definition of compatibility evaluation rules. Furthermore, another characteristics of the service contract compatibility evaluation performed by $\mathrm{SeCO}_{2}$ is that it is not limited to $Q o S$ but it is also extended to other types of property that can be included in a service contract like Business, Service Context and License terms. Table 1 shows the influences between each identified service contract property type and control and data flow.

\begin{tabular}{|l|c|c|c|}
\hline & control flow & data flow & independent \\
\hline Quality of Service $(Q \circ S)$ & $\mathrm{X}$ & & \\
\hline Service Context & & & $\mathrm{X}$ \\
\hline Business & $\mathrm{X}$ & $\mathrm{X}$ & \\
\hline License & $\mathrm{X}$ & $\mathrm{X}$ & \\
\hline
\end{tabular}

Table 1. Data and control flows in contract compatibility evaluation

\section{Modeling and Mapping Service Contract Specifications}

The first step in order to achieve the service contract compatibility is that we have to develop techniques to map different service contracts described in different specifications and terminologies. In our view, the mapping of service contract specifications are not a static, but a dynamic process because specifications and terminologies as well as knowledge about them change over the time. 


\subsection{Typology of Contract Specifications}

Starting from the analysis of ODRL-S, WSLA and WSOL we have identified three types of languages for the specification of service contract properties:

- Type A: includes languages allowing the specification of predefined properties. In this type, e.g., ODRL-S, the properties that can be specified are known by the Language Expert.

- Type B: includes languages allowing the specification of user-defined properties. In this type, e.g., WSLA, the Language Expert knows only the structure of the specification but the properties are defined by the Service Provider.

- Type C: includes languages allowing the specification of properties defined in user ontologies. In this type, e.g., WSOL, the Language Expert knows only the structure of the specification while the properties are specified by the Service Provider using external ontologies.

We use the SeCO Reference Ontology for mapping different specifications and for allowing compatibility evaluation. This ontology is composed of: (i) a core part containing the specification of common properties (e.g., QoS) and (ii) a plug-in part that can be enriched by Language Experts with new properties.

Languages in Type A (e.g., ODRL-S) are characterized by profile models describing all the properties that can be included in a service contract. In this case, the Language Expert enriches the plug-in part to model all the properties not included in the ontology. Moreover, the Language Expert can define fixed mapping rules between properties and ontological concepts.

Languages in Type B (e.g., WSLA) allow new properties to be defined. This characteristic limits the possibility to perform the modeling and mapping of properties in these languages into $\mathrm{SeCO}_{2}$ in advance. Thus, interactions to the Service Providers are still needed when wrapping a concrete service contract into SeCO policies. However, users of the same domain (e.g., the logistic operator domain) typically utilize common terminologies, e.g., logistic operator service providers utilize the term Shipping Location in their specifications which has the same meaning of the property Service Delivery Location available in the core part of the SeCO Reference Ontology. Common terminologies and domain-specific knowledge are used by Language Experts and Domain Experts to define customized mapping rules which will reduce the interactions needed for the wrapping of service contracts.

Languages in Type C (e.g., WSOL) are similar to the ones in Type B but here the properties are semantically described in external ontologies. Thus, the possibility to perform the modeling and mapping activities is limited and we need to define customized mapping rules between concepts in the most common user ontologies and concepts specified in the SeCO Reference Ontology.

Since contract specifications use different representations, ontology alignment tools [11-13], which supports mappings between concepts defined in different ontologies, cannot be used to fully automate the mapping between different specifications. Furthermore, as the interpretation of contract terms may vary from different service providers, fully automatically generation of mapping rules 
cannot be achieved. However, these tools can support the definition of mapping rules when we deal with ontology-based specifications. In this paper, we consider the use of these tools as external activities triggered by the user of $\mathrm{SeCO}_{2}$.

\subsection{Modeling and Mapping Service Contract Terminologies Into the Reference Ontology}

When an XML-based profile model defining properties is available (i.e., Languages in Type A), a set of general rules is used to extract properties from XML-based specifications and semantically describe them into the SeCO Reference Ontology. General rules link an XML-structure to a proper PCM-based description. The same structure can be associated with several rules because also the nature of the property must be considered. Examples are: (i) the XMLstructure in which an element $C 1$ has a set of sub-elements can be linked to the InstanceOf-rule that consider each sub-element as possible values assumed by C1; (ii) the XML-structure in which different elements (e.g., C2 and C3) have the same sub-element $C 1$ is linked to the IsA-rule that considers $C 2$ and $C 3$ as specializations (i.e., sub-concepts) of $C 1$.

To illustrate the above-mentioned techniques, we focus on modeling and mapping ODRL-S terminology. Figure 2 shows how general rules can be used to model ODRL-S properties into the SeCO Reference Ontology. The following ODRL-S terms [4] are considered: (i) Permission Rights: defines types of uses of the service, such as Adaptation, Composition and Derivation; (ii) Payment: describes the financial terms assuming values, such as PrePay and PostPay.

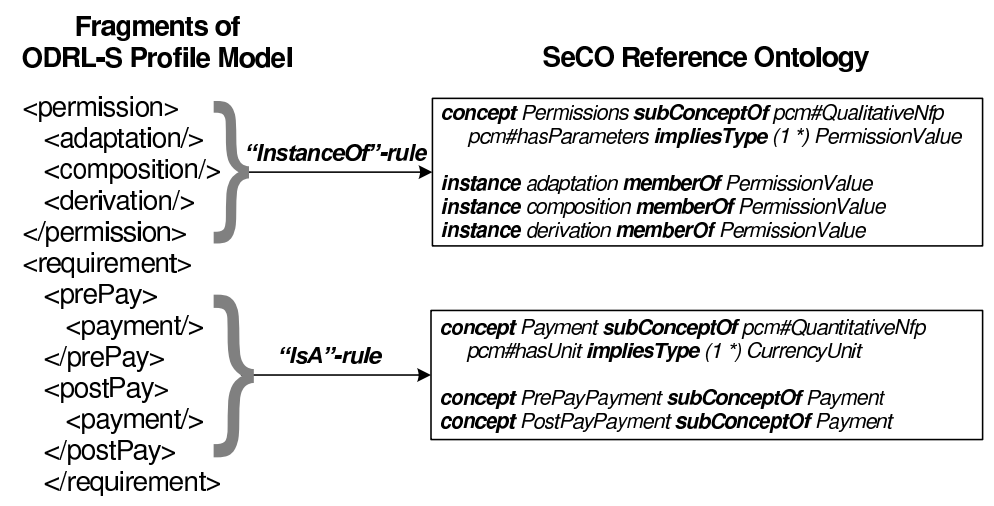

Fig. 2. Modeling ODRL-S properties in the SeCO Reference Ontology

In ODRL-S, Adaptation, Composition and Derivation are sub-elements of Permission. For this property the ODRL-S Language Expert uses the InstanceOfrule to define a new concept Permissions in the ontology which can assume a fixed set of values (i.e., pcm\#hasParameters impliesType PermissionValue) that are Adaptation, Composition and Derivation. PrePay and PostPay are super-elements for Payment. In this case the IsA-rule is applied considering them 
as specializations of the term Payment. A new concept Payment and two subconcepts (PrePayPayment and PostPayPayment) are added to the ontology.

After the modeling of a property in the SeCO Reference Ontology, the Language Expert defines a mapping rule between the property and the related ontological concept; the rule is used in the wrapping of service contract specifications to SeCO Policies. Moreover, the Language Expert stores information into the Contract term knowledge-base about the influences of the property on the data and control flows of the composition. This information is used by Domain Experts for the definition of the related compatibility evaluation rule.

\subsection{Wrapping Service Contract Specifications to SeCO Policy}

A proper technique for each type of language must be defined to perform the wrapping from service contracts to SeCO Policy specifications. The wrapping of specifications in Type A language is directly performed by applying the mapping rules defined by Language Experts. For what concern specifications in Type B and Type $\mathrm{C}$ languages the wrapping activity may require interactions with the Service Providers to handle the absence of knowledge (i.e., mapping rules) on specified properties. The Service Providers must define the mapping between their properties (i.e., text labels for Type B and ontological concepts for Type C) and concepts available in the SeCO Reference Ontology.

For what concern specifications in Type B languages, lexical databases like WordNet support Service Providers to define mapping rules identifying synonyms between text labels and ontological concepts defined in the SeCO Reference Ontology. Different types of ontology alignment tools can be also used to support the wrapping of specifications in Type C languages: (i) tools for defining a mapping between concepts in two different ontologies by finding pairs of related concepts (e.g., ANCHORPROMPT [11]) or by evaluating semantic affinity between concepts (e.g., H-MATCH [12]) and (ii) tools for defining mapping rules to relate only relevant parts of the source ontologies (e.g., ONION [13]).

In this section, we describe a solution for the wrapping of a WSLA specification. The procedure used by $\mathrm{SeCO}_{2}$ is the following: (i) parse the specification in order to detect properties (i.e., SLAParameters); (ii) search the availability of customized mapping rules related to the detected properties; (iii) if mapping rules are not identified, use WordNet to identify a possible mapping between the SLAParameters and concepts available in the SeCO Reference Ontology and ask confirmation about the correctness of the mapping to the Service Provider; and (iv) if the mapping is not correct or not available, ask to the Service Provider to perform the mapping manually.

Figure 3 illustrates the above-mentioned steps when wrapping a WSLAbased service contract consisting PrePayment $=9.99$ Euros and ServiceUsage = ' 'adaptation' '. In this example, a customized mapping rule for PrePayment is identified. On the contrary, the term ServiceUsage is not known and no rules are available. Moreover, no synonym relations are specified in WordNet between ServiceUsage and terms defined in the SeCO Reference Ontology. In order to handle this absence of knowledge, the Service Provider is asked to navigate the 
ontology and map the SLAParameter ServiceUsage to any ontological concept. The result is the mapping of ServiceUsage with Permissions.

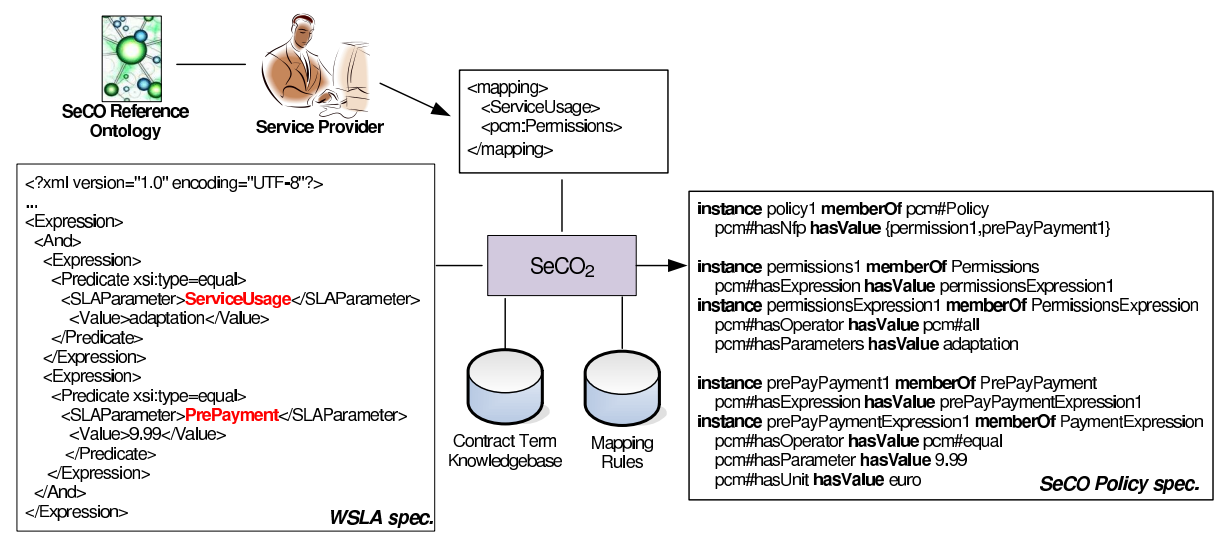

Fig. 3. Mapping between WSLA and SeCO Policy

After this preliminary step, the mapping proceeds considering the Expressions defined in each Service Level Objective of the WSLA specification. Each Expression follows the first order logic, including predicates and logic operators. According to the logic operators, different mapping rules can be applied. The simplest form of a logic expression is a plain predicate. The mapping to a SeCO policy includes the following steps: (i) the mapping rule is used to identify in the SeCO Reference Ontology the concept related to the SLAParameter specified by the Service Provider; (ii) a new instance of this concept is created. It must be characterized by an expression having constraint operator and parameter equals to Type and Value of the Service Level Objective; (iii) a new SeCO Policy containing the concept instance is created.

In Figure 3, the logic operator "And" is used to specify the aggregation of two plain predicates stating conditions on PrePayment and ServiceUsage. The mapping to a SeCO Policy consists in defining the concept instances related to all the plain predicates. The final result for the considered example is a SeCO Policy containing: (i) an instance of Permissions characterized by an expression stating that the value adaptation is assumed (i.e., pcm\#hasOperator hasValue pcm\#all; pcm\#hasParameters hasValue adaptation) and (ii) an instance of PrePayPayment stating that the amount is equal to 9.99 Euros (i.e., pcm\#hasOper ator hasValue pcm\#equal; pcm\#hasParameter hasValue 9.99; pcm\#hasUnit hasValue euro).

\section{Contract Compatibility Evaluation for Service Composition}

The service contract compatibility evaluation supported by the $\mathrm{SeCO}_{2}$ framework accepts a full or part of a full description of service compositions, e.g., the complete structure of a composite service or a workflow region. 


\subsection{Contract Compatibility Evaluation Rules}

The evaluation of service contract compatibility is based on rules defined for service contract properties. As described in [10], service contract properties can be classified into qualitative and quantitative properties. Moreover, as shown in Table 1, properties can differently influence control and data flows.

Qualitative properties must be evaluated considering the relations stored in the SeCO Reference Ontology. Examples of compatibility evaluation rules are:

- "Relation-based" rule: it is applicable to properties assuming values characterized by semantic relations among them. Examples are partnership (i.e., values characterized by part $O f$ relations) and subsumption (i.e., values characterized by is $A$ relations). These relations are checked to verify the compatibility among values associated to a property.

- "Compatible value list" rule: it is applicable to properties assuming a small set of possible values. The compatibility list among these values is stored into the reference ontology by the definition of isCompatibleWith relations.

Quantitative properties must be evaluated considering the constraint operators used to specified the offered values. As described in [10], constraint operators can be binary (e.g., $=, \leq, \geq$ ) or ternary (e.g., range of values). "Binary operator" and "Ternary operator" rules (see [14] for details) evaluate a numeric values in the range [0..1] stating the degree of compatibility between two offered values and the overlap between ranges of values respectively.

\begin{tabular}{|l|c|c|c|c|}
\hline Property & Type & Data Flow & Control Flow & Rule \\
\hline Service Delivery Location & Service Context & & & partnership \\
\hline Pricing & Business & $\mathrm{X}$ & & compatible value list \\
\hline Payment (for data usage) & Business & $\mathrm{X}$ & & binary, ternary \\
\hline Payment (for service usage) & Business & & $\mathrm{X}$ & binary, ternary \\
\hline Scalability & QoS & & $\mathrm{X}$ & binary, ternary \\
\hline Request Limit & QoS & & $\mathrm{X}$ & binary, ternary \\
\hline Availability Time Range & QoS & & $\mathrm{X}$ & ternary \\
\hline Data Ownership & License & $\mathrm{X}$ & & compatible value list \\
\hline Permissions & License & & $\mathrm{X}$ & subsumption \\
\hline
\end{tabular}

Table 2. Examples of common rules

Table 2 presents some common rules for the evaluation. We explain some of them in the following. The Service Delivery Location property is independent from data and control flows since its value must be checked in all the contracts of the services involved in the composition. The compatibility is evaluated applying a "Relation-based" rule focusing on partnership relations ( $\sqsupseteq)$. In particular, services $s_{1}$ and $s_{2}$ are compatible if $s_{1}$.value $\sqsupseteq s_{2}$.value or $s_{2}$.value $\sqsupseteq s_{1}$.value. For example, let us assume that $s_{1}$ delivers in the Worldwide, $s_{2}$ in Europe and $s_{3}$ in the US. The following partnership relations are hold: Worldwide $\sqsupseteq$ Europe and Worldwide $\sqsupseteq$ US. Thus, services $s_{2}$ and $s_{3}$ cannot be included in the same composition since their provision is limited to different geographical area. 
The compatibility on Pricing terms in service contracts is checked considering the data flow. The evaluation is performed using a "Compatible value list" rule stating the compatibility among possible pricing models. For example, flat rate is compatible with pay per use with subscription but it is incompatible with free per use.

The property Scalability is checked considering the composition patterns included in the control flow specification and applying a "Binary operator" rule. For example, assume that service $s_{1}$ and $s_{2}$ follow a sequential execution and that $s_{1}$ and $s_{2}$ have Scalability $=s c_{1}$ and Scalability $=s c_{2}$, respectively. Services $s_{1}$ and $s_{2}$ are compatible if $s c_{1} \leq s c_{2}$.

\subsection{An Algorithm for Contract Compatibility Evaluation}

Let $S=\left\{s_{1}, s_{2}, \cdots, s_{m}\right\}$ denote the set of services involved in the composition. Each service is characterized by a service operation associated with one or more SeCO Policies. Let $P\left(s_{i}\right)=\left\{p_{1}, p_{2}, \cdots, p_{n}\right\}$ indicate the set of policies associated to service $s_{i}$. Each policy is composed of one or more offered properties. Let $P R\left(p_{i}\right)=\left\{p r_{1}, p r_{2}, \cdots, p r_{w}\right\}$ be the set of properties offered by policy $p_{i}$. Each property is specified by: (i) a name stating the related ontological concept; (ii) a type defining if the property is CF-inf (i.e., influence the control flow), DF-inf (i.e., influence the data flow) or F-ind (i.e., flow independent); (iii) an operator; (iv) a value and (v) a unit of measure.

Let $C F\left(s_{i}\right)=\left\{c f_{1}, c f_{2}, \cdots, c f_{m}\right\}$ denote the control flow where each $c f_{j}$ in $C F\left(s_{i}\right)$ specifies the composition pattern between $s_{i}$ and $s_{j}$. Possible values are sequential, parallel and conditional execution. Let $D F\left(s_{i}\right)=\left\{d f_{1}, d f_{2}, \cdots, d f_{m}\right\}$ denote the data flow where each $d f_{j}$ in $D F\left(s_{i}\right)$ specifies if there is a dependency in data provisioning between $s_{i}$ and $s_{j}$.

Our service contract compatibility algorithm is listed in Algorithm 1. The algorithm evaluates the compatibility among all the policies of all the couples of services available in the composition. Line 3 defines $\Omega\left(s_{i}, s_{j}\right)$ as a set of triples. Each triple will contain a policy $p_{w}$ associated to $s_{i}$, a policy $p_{z}$ associated to $s_{j}$, and the result of the compatibility evaluation $\lambda\left(p_{w}, p_{z}\right)$ among them. The evaluation of $\lambda\left(p_{w}, p_{z}\right)$ starts in Line 7 defining $\Upsilon\left(p_{w}, p_{z}\right)$ as a set of comparable properties $\left[p r_{1}, p r_{2}\right]$ specified in $p_{w}$ and $p_{z} . \Upsilon\left(p_{w}, p_{z}\right)$ is populated by the Matching procedure (Line 8) that applies matching rules similar to the ones shown in [10]. For each identified couple $\left[p r_{1}, p r_{2}\right]$ of comparable properties, the algorithm retrieves the related evaluation rule using the procedure Extract and specifying the property name (Line 10). As stated above, at this point the evaluation proceeds considering the property type. If the property is $C F$-inf then procedure EvalRuleF is invoked specifying the retrieved rule, the two comparable properties $\left[p r_{1}, p r_{2}\right]$ and the control flow information about the services $s_{i}, s_{j}$ that offer the properties (Line 12). If the property is DF-inf then the same procedure EvalRuleF is invoked but specifying the data flow information about the services $s_{i}, s_{j}$ (Line 15). Finally, if the property is $F$-ind then the procedure EvalRule that does not consider composition flows is invoked (Line 17). The result of the evaluation is saved in $\lambda\left(p_{w}, p_{z}\right)$ that contains the evaluation of all the 


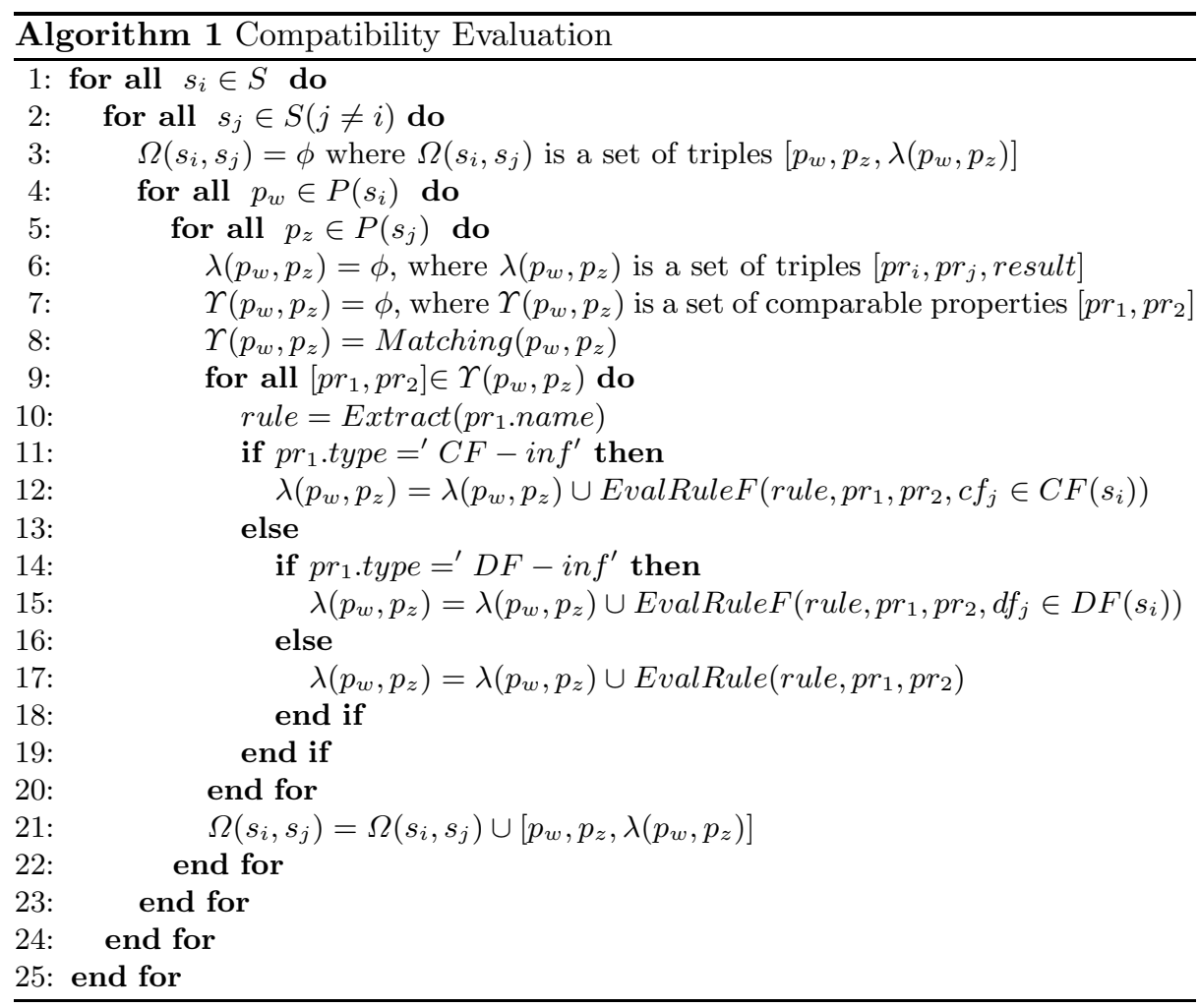

comparable properties in $p_{w}$ and $p_{z}$. Finally, the triple $\left[p_{w}, p_{z}, \lambda\left(p_{w}, p_{z}\right)\right]$ is saved in $\Omega\left(s_{i}, s_{j}\right)$ (Line 21) that contains the evaluation for all the policies offered by $s_{i}$ and $s_{j}$.

\section{$6 \quad$ Illustrating Scenarios}

In order to demonstrate the contract compatibility evaluation techniques proposed in Section 5, we consider a process of purchase data analysis inside a supply chain management scenario. This process involves multiple services collaborating with each others: (i) a Request Service (RS) issuing a purchase request; (ii) a Purchase Processing Service (PPS) managing the standard e-commerce process; (iii) a Merchant Validation Service (MVS) verifying and providing data about a shopping merchant; (iv) a Payment Verification Service (PS) validating data related to the payment (e.g., the credit card number); (v) Shipping Evaluation Service (SES) calculating shipping charges and (vi) a Purchase Validation Service (PVS) analyzing data and validating the purchase. These services can be composed using different control and data flows. Figure 4 shows two different possible composition structures. 

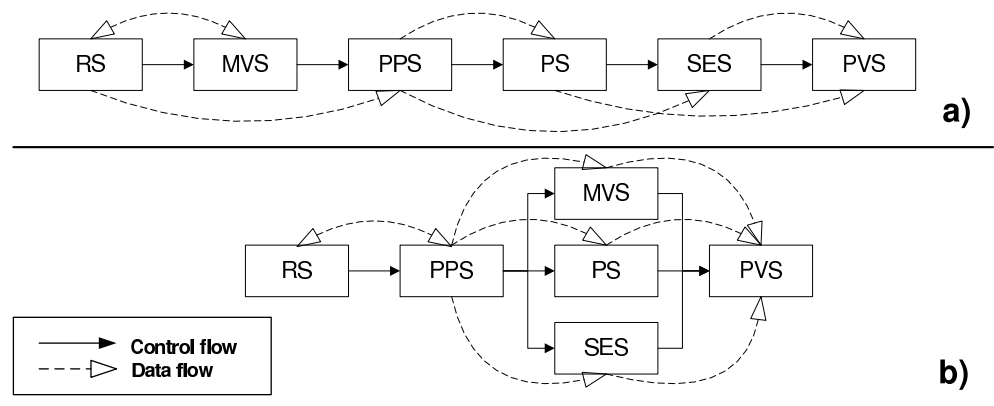

Fig. 4. Different composition structures for the Purchase Data Analysis service.

Let us assume that a service consumer wants to create a Purchase Data Analysis (PDA) service by composing his/her RS with the following Web services: (i) Yahoo! Shopping Web Service ${ }^{5}$ as MVS; (ii) XWebCheckOut Web Service $^{6}$ as PPS; (iii) Aivea Shipping Web Service ${ }^{7}$ as SES; (iv) ValidateCreditCard Web Service ${ }^{8}$ as PS and (v) DOTS Lead Validation Web Service ${ }^{9}$ as PVS. For our experiments, we focus only on service contracts. Thus, let us assume that these Web services match the functionalities required for the PDA service.

The selected Web services are characterized by service contracts available only as HTML texts in their Websites (i.e., ODRL-S, WSLA and WSOL specifications are not available). Moreover, these contracts are unclear, ambiguous and limited to few information. This forces the service consumer to manually compare them and, often, further information from the service providers are needed. Modeling and mapping techniques presented in Section 4 are not applicable due to the absence of structured specifications. In order to overcome this strong limitation, we produce SeCO Policies using information described in the HTML texts and inserting realistic properties in case of limited descriptions. These policies are summarized in Table 3. For each selected Web service, we consider the properties Service Delivery Location, Pricing and Scalability described in Section 5. Moreover, we consider Data Ownership (a license term stating how the data produced by the service are protected) and Request Limit (a license term defining the maximum number of requests that a user can submit to the service in a day).

Applying evaluation rules like the ones described in Section 5, the compatibility evaluation results produced by our $\mathrm{SeCO}_{2}$ framework are given in Figures 5 and 6. The following results must be underlined: (i) incompatibility on Service Delivery Location is found in both the compositions since the property is independent from data and control flows; (ii) incompatibility on Pricing is found in both the compositions because both are characterized by a data flow from RS and

\footnotetext{
${ }^{5}$ http://developer.yahoo.com/shopping/V1/merchantSearch.html

${ }^{6}$ http://www.xwebservices.com/Web_Services/XWebCheckOut/

${ }^{7}$ http://www.aivea.com/shipping-web-service.htm

${ }^{8} \mathrm{http}: / /$ www.webservicex.net/WCF/ServiceDetails.aspx?SID $=14$

${ }^{9} \mathrm{http}: / /$ www.serviceobjects.com/products/composite/lead-validation
} 


\begin{tabular}{|l|c|c|c|c|c|}
\hline & Del.Loc. & Data Own. & Request Limit & Pricing & Scalability \\
\hline Request Service $(R S)$ & US & personal-use & unlimited & free & $100 \mathrm{tr} / \mathrm{min}$ \\
\hline Yahoo! Shopping $(M V S)$ & Worldwide & copyrighted & $5000 \mathrm{q} /$ day & free & $100 \mathrm{tr} / \mathrm{min}$ \\
\hline XWebCheckOut $(P P S)$ & Worldwide & free-distrib. & unlimited & $100 \$ /$ year & $100 \mathrm{tr} / \mathrm{min}$ \\
\hline Aivea Shipping $(S E S)$ & Europe & free-distrib. & unlimited & $49 \$ / \mathrm{month}$ & $100 \mathrm{tr} / \mathrm{min}$ \\
\hline ValidateCreditCard $(P S)$ & Worldwide & free-distrib. & unlimited & free & $500 \mathrm{tr} / \mathrm{min}$ \\
\hline DOTS Lead Valid. $(P V S)$ & Worldwide & free-distrib. & unlimited & free & $500 \mathrm{tr} / \mathrm{min}$ \\
\hline
\end{tabular}

Table 3. Contracts offered by services involved in the composition

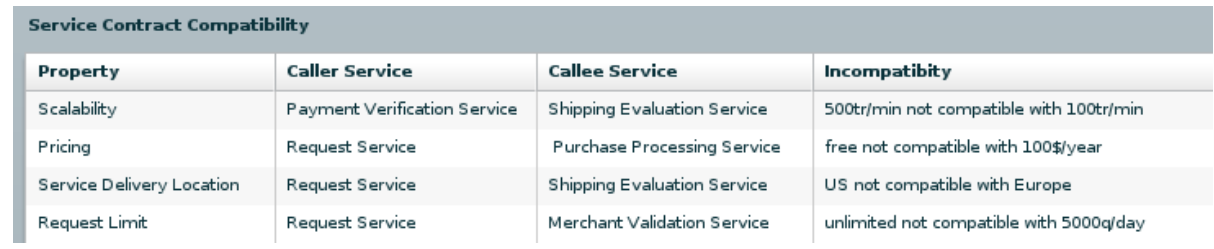

Fig. 5. Resulting compatibility evaluation for Composition a (Figure 4(a))

\begin{tabular}{|l|l|l|l|}
\hline \multicolumn{2}{|l}{ Service Contract Compatibility } & & \\
\hline Property & Caller Service & Callee Service & Incompatibity \\
\hline Data Ownership & Merchant Validation Service & Purchase Validation Service & copyrighted not compatible with free-distribution \\
\hline Pricing & Request Service & Purchase Processing Service & free not compatible with 100\$/year \\
\hline Request Limit & Purchase Processing Service & Merchant Validation Service & unlimited not compatible with 5000q/day \\
\hline Service Delivery Location & Request Selaste & Shipping Evaluation Service & US not compatible with Europe \\
\hline
\end{tabular}

Fig. 6. Resulting compatibility evaluation for Composition b (Figure 4(b))

PPS; (iii) incompatibility on Request Limit is found in both the compositions but between different services. This is determined by the different data flows involving MVS; (iv) incompatibility on Scalability is found only in composition a. This result depends on the different control flows (i.e., in composition a SES is invoked after PS instead in composition b it is invoked after PPS); (v) incompatibility on Data Ownership is found only in composition b. This result depends on the different data flows (i.e., in composition a MVS data are managed by RS instead in composition $b$ they are managed by PVS).

\section{Concluding Remarks}

In this paper, we have presented our approach to checking service contract compatibility for service compositions. Our $\mathrm{SeCO}_{2}$ framework provides support to define, update, and share knowledge about service contracts specified by different specifications. Our work can map different service contracts and determined the compatibility based on control and data flows, as well as composition patterns.

Our approach is currently tested with ODRL-S, WSLA, and WSOL. There is no way to automatically determine the typology of a language, thus mapping rules still involve domain experts. We think that it is inevitable, unless terminologies are well-defined and agreed by all service providers. Currently, we do not consider the dynamic changes of contracts during the composition. For example, when performing the composition, the customer and service providers might negotiate the contracts, as the contract changes certain steps have to be rerun. 
However, currently we consider this change can be solved only by re-running the compatibility checking. Our future work includes enhancing this dynamic interaction among actors when dealing with service contracts. Furthermore, data specific contract compatibility will be improved.

\section{References}

1. Armbrust, M., Fox, A., Grifth, R., Joseph, A.D., Katz, R., Konwinski, A., Lee, G., Patterson, D., Rabkin, A., Stoica, I., Zaharia, M.: Above the clouds: A berkeley view of cloud computing. Technical report, University of California at Berkeley (2009)

2. Truong, H., Gangadharan, G., Treiber, M., Dustdar, S., D'Andrea, V.: On reconciliation of contractual concerns of web services. In: In NFPSLASOC'08 (2nd Non Functional Properties and Service Level Agreements in SOC Workshop), Dublin, Ireland (2008)

3. Gangadharan, G.R., Weiss, M., D'Andrea, V., Iannella, R.: "Service License Composition and Compatibility Analysis". In: "Proceedings of the International Conference on Service Oriented Computing (ICSOC'07), Vienna, Austria". (2007)

4. Gangadharan, G.R., D'Andrea, V., Iannella, R., Weiss, M.: "ODRL Service Licensing Profile (ODRL-S)". In: "Proceedings of the 5th International Workshop for Technical, Economic, and Legal Aspects of Business Models for Virtual Goods". (2007)

5. Ludwig, H., Keller, A., Dan, A., King, R., Franck, R.: "Web Service Level Agreement (WSLA) Language Specification". IBM Coporation (2003)

6. Tosic, V., Pagurek, B., Patel, K., Esfandiari, B., Ma, W.: "Management Applications of the Web Service Offerings Language (WSOL)". Information Systems 30(7) (2005) 564-586

7. Zeng, L., Benatallah, B., Ngu, A., Dumas, M., Kalagnanam, J., Chang, H.: Qosaware middleware for web services composition. IEEE Trans. Softw. Eng. 30(5) (2004) 311-327

8. Jaeger, M., Rojec-Goldmann, G., Muhl, G.: Qos aggregation for web service composition using workflow patterns. In: EDOC '04: Proceedings of the Enterprise Distributed Object Computing Conference, Eighth IEEE International, Washington, DC, USA, IEEE Computer Society (2004) 149-159

9. Aggarwal, R., Verma, K., Miller, J., Milnor, W.: Constraint driven web service composition in meteor-s. In: Proceedings of the 2004 IEEE International Conference on Services Computing (SCC 2004). (2004) 23-30

10. De Paoli, F., Palmonari, M., Comerio, M., Maurino, A.: A Meta-Model for NonFunctional Property Descriptions of Web Services. In: Proceedings of the IEEE International Conference on Web Services (ICWS), Beijing, China (2008)

11. Noy, N.F., Musen, M.A.: The prompt suite: Interactive tools for ontology merging and mapping. International Journal of Human-Computer Studies 59 (2003) 2003

12. S. Castano, A. Ferrara, S.M.: H-match: an algorithm for dynamically matching ontologies in peer-based systems. In: Proc. of the 1st VLDB Int. Workshop on Semantic Web and Databases (SWDB 2003), Berlin, Germany (2003)

13. Mitra, P., Wiederhold, G., Decker, S.: A scalable framework for the interoperation of information sources. In: Stanford University. (2001) 317-329

14. Comerio, M., De Paoli, F., Maurino, A., Palmonari, M.: "NFP-aware Semantic Web Services Selection". In: "Proceedings of the 11th IEEE International Enterprise Distributed Object Computing Conference (EDOC)". (2007) 